\title{
Sistematização da assistência de enfermagem - proposta de diagnóstico de enfermagem específico para o paciente idoso com doença cardiovascular
}

\author{
Kelly Regina Lainetti*, Márcia de Fátima da Silva, M.Sc.**
}

*Enfermeira, Curso de Enfermagem Cardiovascular do Instituto Dante Pazzanese de Cardiologia (IDPC),

** Enfermeira, Escola de Enfermagem da Universidade de São Paulo (EEUSP)

\begin{abstract}
Resumo
Trata-se de uma pesquisa quali-quantitativa, descritiva e exploratória, que teve como objetivo levantar os problemas de enfermagem dos pacientes portadores de patologia cardiovascular no intuito de descrever os diagnósticos de enfermagem específicos a esta populaçáo. Foram abordados 84 pacientes com idade igual ou maior de 60 anos e levantados os reais problemas de enfermagem apresentados nestes pacientes durante o período de janeiro a março de 2008. Os resultados foram analisados obtendo o levantamento de 23 diagnósticos específicos para esta população de acordo com os problemas encontrados. O estudo permitiu apontar algumas contribuições para a área assistencial por identificar os problemas e levantar os diagnósticos de enfermagem específicos a esta população, o que contempla a atitude do enfermeiro como instrumento na ação do cuidado ao paciente idoso.
\end{abstract}

Palavras-chave: idoso, doenças cardiovasculares, diagnóstico de enfermagem.

\section{Abstract \\ Nursing assistance systematization - proposal of nursing diagnosis specific for the elderly patient with cardiovascular disease}

This quali-quantitative study with exploratory and descriptive design aims at finding out nursing problems related to patients with cardiovascular pathology in order to describe specific nursing diagnosis for the elderly. From January to March 2008, 84 patients, aged 60 or more, were selected to search for real nursing problems with these patients. The results were analyzed and 23 specific diagnoses for this population were obtained. The study allows to point out some contributions to care area, identifying problems and finding out specific nursing diagnoses for this population and to consider the nurses attitude as instrument of care for elderly patients.

Key-words: aged, cardiovascular disease, nursing diagnosis. 


\section{Resumen}

\section{Sistematización de la asistencia de enfermería - propuesta de diagnóstico de enfermería específico para el paciente mayor con enfermedad cardiovascular}

Se trata de una investigación cuali-cuantitativa, descriptiva y exploratoria, que tuvo como objetivo identificar los problemas de enfermería en los pacientes portadores de patología cardiovascular con la intención de describir los diagnósticos de enfermería específicos en esta población. Fueron abordados 84 pacientes de edad igual o superior a 60 años e identificado los problemas reales de enfermaría encontrados en estos pacientes durante el período de Enero a Marzo del 2008. Los resultados fueron analizados y se dieron a conocer 23 diagnósticos específicos para ésta población de acuerdo con los problemas encontrados. El estudio permitió aportar algunas contribuciones para el área asistencial al identificar los problemas específicos en ésta población, lo que contempla la actitud del enfermero como instrumento en la acción al cuidado del paciente mayor.

Palabras-clave: anciano, enfermedades cardiovasculares, diagnóstico de enfermería.

\section{Introdução}

O envelhecimento afeta todas as estruturas e funçôes do corpo caracterizando-se como um processo heterogêneo $[1,2]$. O organismo humano, desde sua concepção até a morte, passa por diversas fases: desenvolvimento, puberdade, maturidade ou de estabilização e envelhecimento [3].

A gerontologia trata do processo de envelhecimento em seus aspectos preventivo, clínico, terapêutico, de reabilitação e de vigilância contínua. Enfoca a biofisiologia, a genética, a imunologia e o envelhecimento a níveis celular e subcelular. Para tanto existe ainda a gerontologia social que cuida das leis que protegem os direitos e deveres dos idosos [4]. A expectativa de vida do idoso no mundo está em constante crescimento. Tem-se verificado no Brasil nas últimas décadas, um decréscimo nas taxas de natalidade e mortalidade, o que tem proporcionado um aumento na população de faixa etária entre 60 anos ou mais [5]. A lei 5.145/04, baseada na Legislação Federal do Brasil e no Estatuto dos Idosos de 2004, considera idoso toda pessoa que se encontra na faixa etária a partir dos 60 anos [6]. Segundo o IBGE o número de idosos em 2000 era de aproximadamente 15 milhóes, em 2025 há estimativas que este número alcance 34 milhóes. Dados estatísticos apontam que no Brasil o aumento da população idosa, entre 1950 e 2025, crescerá mais 15 vezes [2,7-9]. Acredita-se que no ano de 2050 os idosos serão um quinto da população mundial [3].

As populaçóes dos países desenvolvidos, que possuem expectativa média de vida de aproximadamente 77 anos, já estão apresentando curvas próximas do contorno retangular. Da mesma forma que nos países em desenvolvimento, com expectativa de vida de 67 anos, já está ocorrendo certa tendência a esta retangularização da curva embora distante do nível atingido pelo primeiro mundo [3]. Esse fenômeno ocorre em parte pela diminuição das taxas de fecundidade, e também, pelo aumento na expectativa de vida das populaçóes necessitando, por conseguinte, de uma adaptação a essa nova realidade $[2,5,9,10]$.

Desde 1963, as doenças cardiovasculares superaram as outras causas de morte, sendo responsáveis atualmente por $27 \%$ dos óbitos [11]. Pesquisadores mostram a ocorrência das mudanças em nível cardíaco. A capacidade do coração de aumentar o número e a força dos batimentos cardíacos diminui quando submetidos a esforços. É comum encontrarem-se extra-sístoles, assim como sopro sistólico mitral. A reserva cardíaca fica rebaixada assim como o consumo de oxigênio devido à fibrose, à rigidez, aos depósitos de gordura, à diminuição na elasticidade das artérias, com conseqüente aumento da pressão sistólica. A pressão arterial diastólica costuma variar pouco [12]. Os diagnósticos médicos de doenças cardiovasculares mais prevalentes nos idosos são: hipertensão arterial sistêmica (42\%), coronariopatia (18\%), eletrofisiologia (14\%), marcapasso cardíaco (13\%), valvulopatia (6\%), cardiopatia congênitas (1\%) [13].

Devemos para tanto, assistir o homem em todas as suas fases da vida, tanto na saúde como na doença com qualidade no cuidado de enfermagem. Para que isso possa acontecer, há necessidade de maior envolvimento nesta especialidade.

Gunter e Miller apud Campedelli [4] definem como enfermagem gerontológica o estudo científico 
do cuidado de enfermagem ao idoso que é caracterizada como a ciência aplicada com propósito de utilizar os conhecimentos dos processos de envelhecimentos, para o planejamento da assistência de enfermagem e dos serviços que melhor atendem à promoção da saúde, à longevidade, à independência e ao nível mais alto possível de funcionamento da pessoa idosa. Diz ainda que a enfermagem geriátrica está relacionada às necessidades de enfermagem das pessoas idosas, ao planejamento e implementação dos cuidados de enfermagem que irão ao encontro dessas necessidades e à avaliação de um nível consistente de bem-estar, com as limitaçóes impostas pelo processo de envelhecimento.

Partindo do pressuposto de que sistematizar é fazer alguma coisa em etapas, fases ou passos; sistematizar a assistência implica lançar mão de uma teoria de enfermagem como parâmetros para fundamentar o agir. Uma teoria quando se torna prática, ação do dia-a-dia, suscita um processo. $\mathrm{O}$ método científico de trabalho do enfermeiro é o processo de enfermagem [14].

A assistência de enfermagem é definida como a aplicaçáo, pelo (a) enfermeiro (a), do processo de enfermagem para prestar o conjunto de cuidados e medidas que visam atender às necessidades básicas do ser humano; e o cuidado de enfermagem como a ação planejada, deliberada ou automática do (a) enfermeiro (a), resultante de sua percepção, observação e análise do comportamento, situação ou condição do ser humano [15].

A assistência de enfermagem baseia-se em uma estrutura lógica de açóes que chamamos de Sistematização da Assistência de Enfermagem (SAE), a qual é composta por etapas e processos, sendo eles: histórico, obtido através de entrevista e exame físico; diagnóstico de enfermagem; prescrição de enfermagem; e evolução de enfermagem. No idoso o exame físico deve ir além da avaliação clínica. Esses pacientes são mais susceptíveis a desenvolver limitaçóes para atividades de vida diária e manifestar as chamadas sindromes geriátricas [16]. Estas síndromes são conhecidas como os grandes "Is" da geriatria: imobilidade, instabilidade, incontinência, insuficiência cerebral, iatrogenia [17].

O diagnóstico de enfermagem foi definido na $9^{\circ}$ conferência da NANDA (North American Nursing Diagnosis Association) como "um julgamento clínico das respostas do indivíduo, da família ou da comunidade aos processos vitais, aos problemas de saúde atuais ou potenciais, os quais fornecem a base para a seleção das intervençóes de enfermagem, para atingir resultados pelos quais o enfermeiro é responsável” [18]. Um diagnóstico de enfermagem consiste em "um julgamento clínico sobre a resposta de um indivíduo, uma família ou uma comunidade com relação a problemas de saúde reais ou potenciais/ processos de vida que fornecem a base para a terapia definitiva que busca alcançar resultados nos quais a enfermagem é necessária” [19].

O diagnóstico de enfermagem específico para o paciente idoso tem como objetivo intervir e prevenir que problemas tornem-se ativos ou limitar suas conseqüências através de intervençóes especificas e apropriadas, de problemas já instalados. A capacitação da máo de obra técnica para atender adequadamente ao idoso pressupóe conhecimentos específicos nas áreas de geriatria e gerontologia. A carência de recursos humanos especializados é um dos grandes problemas observados na inadequação dessa assistência [20].

Campedelli [4] afirma que "os idosos poderão viver mais, se houver prevenção específica de seus problemas".

O presente estudo tem como objetivo abordar os problemas mais freqüentes na população idosa atendida no local de estudo, Instituto Dante Pazzanese de Cardiologia (IDPC), dentre as clínicas de Marcapasso, Hipertensão, Vascular, Congênita, eletrofisiologia, miocardiopatia, válvula e coronária, patologias estas mais freqüentes e mais presentes no paciente idoso.

\section{Material e métodos}

Trata-se de uma pesquisa descritivo exploratório com abordagem quanti-quali, que teve como objetivo identificar os problemas e elaborar os diagnósticos específicos utilizando amostragem estratificada não-proporcional, implicando na seleção de pacientes com idade igual ou superior a 60 anos, portador de doença cardiovascular associada, de ambos os sexos internados no setor de enfermaria de um hospital público de grande porte especializado na área de cardiologia. A coleta se efetivou mediante a realização de exame físico céfalo-caudal e a aplicação de um instrumento semi-estruturado (histórico de enfermagem) já utilizado na instituição, contendo questóes abertas e fechadas. Este foi aplicado no período de fevereiro a abril de 2008 nas primeiras 24 horas a partir da data de admissáo do paciente, após a aprovação do estudo pelo comitê de ética da 
instituição sob número de protocolo FR 148694 e assinatura pelo paciente /responsável do termo de consentimento livre e esclarecido.

\section{Resultados e discussão}

No presente estudo foram entrevistados e examinados fisicamente 98 idosos com idade igual ou superior a 60 anos, internados por patologia cardiovascular, no período de 01 de fevereiro a 30 de abril 2008, apresentando-se mais frequentemente na faixa etária entre 70 a 79 anos (43\%), conforme mostra o Figura 1.

Figura 1 - Faixa etária dos pacientes idosos portadores de doenças cardiovasculares internados no período de 01 fevereiro a 30 de abril. São Paulo, 2008.

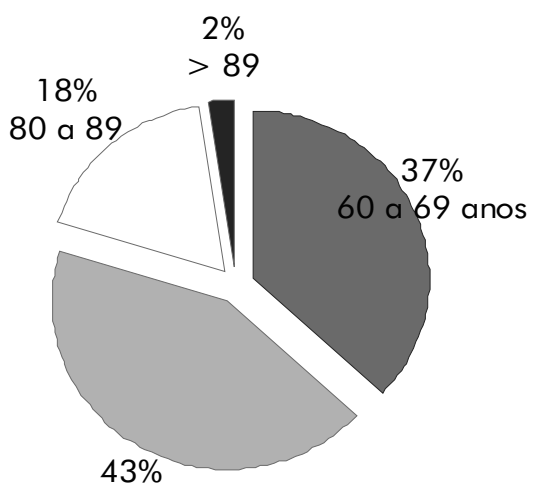

70 a 79 anos

Dentre os 98 pacientes, somente 84 aceitaram participar da pesquisa assinando o termo de consentimento livre e esclarecido. As perdas obtidas (14 pacientes) se deram referentes à: $(43 \%)$ internação por período menor que 24 horas no setor (procedimentos diagnósticos como cateterismo, estudo eletrofisiológico, cirurgias eletivas ou por piora do quadro clínico do paciente, resultando em transferência para outro setor), (22\%) impossibilidade de responder ao histórico de enfermagem por ausência de acompanhante durante o período de coleta dentro das 24 horas propostas pelo estudo, (21\%) por dependência de terceiros para assinar o termo (filhos ou cônjuge), (14\%) por recusar-se a participar da pesquisa. Os sujeitos da pesquisa se constituíram em $57 \%$ do sexo masculino e $43 \%$ do sexo feminino. As patologias foram computadas através das respectivas áreas clínicas de tratamento apresentando-se com maior prevalência respectivamente as clínicas: (26\%) Miocárdio, (21\%) Marcapasso, (17\%) Vascular, (12\%) Eletrofisiologia, (7\%) Coronária, (2\%) Válvula, (2\%) Nefro, (2\%) Hipertensão, (1\%)
Congênito e (10\%) internação por outras complicaçôes hospitalares. Cabe salientar que muitos dos pacientes internados apresentaram mais do que uma patologia, totalizando um número de 121 patologias diagnosticadas e listadas. Segue abaixo, (Tabela I) as patologias mais comuns referentes a cada clínica citada acima:

Tabela I - Patologias mais comuns entre os idosos do estudo.

\begin{tabular}{|c|c|c|}
\hline Clínica & Patologia & $\mathrm{N}$ \\
\hline \multirow{4}{*}{ Miocárdio } & ICC & 17 \\
\hline & MCP & 8 \\
\hline & Chagas & 5 \\
\hline & Bradicardia & 1 \\
\hline \multirow{3}{*}{ Marcapasso } & BAVT & 12 \\
\hline & Sincope AE & 9 \\
\hline & Implante de MP & 5 \\
\hline \multirow{5}{*}{ Vascular } & IAPC & 7 \\
\hline & AAA & 7 \\
\hline & Estenose carótida & 2 \\
\hline & Lesão trófica MMII & 2 \\
\hline & Amputação & 2 \\
\hline \multirow{3}{*}{ Válvula } & Estenose aórtica & 1 \\
\hline & Endocardite & 1 \\
\hline & Troca válvula & 1 \\
\hline \multirow{4}{*}{ Eletrofisiologia } & Arritmia & 6 \\
\hline & FA & 5 \\
\hline & Flutter atrial & 2 \\
\hline & Disfunção CDI & 1 \\
\hline \multirow{2}{*}{ Coronária } & SCA & 6 \\
\hline & $\mathrm{ICO}$ & 3 \\
\hline Nefrologia & IRC & 3 \\
\hline Hipertensão & Hipertensão & 2 \\
\hline Congênito & Anomalia de Ebstein & 1 \\
\hline \multirow{7}{*}{$\begin{array}{l}\text { Outras patologias/ } \\
\text { complicações }\end{array}$} & TVP & 4 \\
\hline & TEP & 3 \\
\hline & Edema agudo pulmão & 1 \\
\hline & ITU & 1 \\
\hline & DPOC & 1 \\
\hline & Erisipela & 1 \\
\hline & $\mathrm{BCP}$ & 1 \\
\hline
\end{tabular}

Legenda: ICC (Insuficiência cardíaca congestiva), MCP (Miocardiopatia), BAVT (Bloqueio átrio-ventricular total), AAA (Aneurisma de Aorta Abdominal), FA (Fibrilação atrial), Disfunção de CDI (Disfunção do Cardiodesfibrilador Implantável), SCA (Síndrome coronariana aguda), ICO (Insuficiência coronariana), IRC (Insuficiência renal crônica), BCP (Broncopneumonia), ITU (Infecção trato urinário), DPOC (Doença pulmonar obstrutiva crônica), TEP (Trombo embolismo pulmonar), TVP (Trombose venosa profunda). 
Para levantamento dos dados foi utilizado o histórico de enfermagem já aplicado rotineiramente na instituição. Para tanto, nos pacientes que já tinham histórico de enfermagem de internações passadas, este foi examinado novamente e teve seu histórico de enfermagem atualizado pela pesquisadora. Por ser o histórico de enfermagem um documento legal do paciente e da instituição onde o mesmo se encontra internado, foi necessária a criação de um instrumento facilitador de coleta de dados, onde foi possível transcrever os dados contidos no histórico de enfermagem para posterior coleta e tabulação destes dados fora do âmbito hospitalar, fazendo com que este documento permanecesse sempre junto ao prontuário do paciente.

Partindo-se do referencial teórico de que problemas de enfermagem são aqueles em que se intervindo podemos solucioná-los, foram encontrados diversos problemas de enfermagem de acordo com a patologia diagnosticada pelo médico [15].

Para o possível levantamento dos diagnósticos de enfermagem foram tabuladas as alteraçóes (problemas de enfermagem) encontradas através da entrevista e do exame físico realizado nestes pacientes, referentes a cada sistema do organismo, respeitando a ordem céfalo-cadal. Para tanto, os diagnósticos de enfermagem, específicos ao paciente idoso portador de cardiopatia e/ou doença vascular, foram levantados baseados na apresentação das alteraçóes encontradas (problemas de enfermagem) e demonstradas com maior freqüência, para caracterizar o diagnóstico levantado e apresentar os necessários fatores relacionados para concretizar o diagnóstico prescrito.

Com base nos problemas de enfermagem encontrados foram levantados os seguintes diagnósticos específicos de enfermagem:

- Risco de quedas - fator de risco: história de queda (medo), uso de aparelho ou artefato (bengala, muleta, andador, cadeira de rodas), idade de 65 anos ou mais, morar sozinho, insônia, doença vascular, marcha afetada (acamado, dificuldade para deambular), equilíbrio prejudicado (tontura). Ansiedade relacionada à morte - caracterizada por: tristeza, insônia, medo de morrer. Relacionado ao desconhecimento da patologia/ terapêutica.

- Débito cardíaco diminuído - caracterizado por: arritmia (ritmo irregular), alteração no eletrocardiograma, edema, oligúria, pulsos periféricos fracos/ausentes. Relacionado à tosse, à dispnéia, à ansiedade, à contratilidade cardíaca alterada (falha em bomba cardíaca).

- Perfusão tissular periférica ineficaz - caracterizada por: edema, pulsos periféricos fracos/ausentes, descoloraçáo da pele (MMII descorados). Relacionado à interrupção do fluxo arterial.

- Integridade da pele prejudicada - caracterizada por: desnutriçáo, rompimento das camadas da derme/epiderme (lesôes). Relacionado à marcha afetada (acamado, à dificuldade para deambular), à idade avançada (60 anos ou mais), ao estado nutricional alterado (obesidade, emagrecimento), à circulação alterada (MMII descorados), à alteração no turgor (mudanças na elasticidade), ao desequilíbrio líquido (edema, desidratação).

- Integridade tissular prejudicada - caracterizada por: tecido lesado ou destruído (amputação, tecido desvitalizado). Relacionado ao: déficit nutricional (inapetência, ao IMC alterado), ao déficit de conhecimento (desconhece a patologia/terapêutica), à marcha afetada (acamado, dificuldade para deambular), à circulação alterada (pulsos periféricos fracos/ ausentes, varizes MMII, MMII descorados), déficit ou excesso de líquidos (desidratação/ edema).

- Desobstrução ineficaz de vias aéreas - caracterizada por: dispnéia, freqüência e ritmo respiratório irregular, ruídos adventícios respiratórios (sibilos, roncos e crepitação). Relacionado ao tabagismo e à dificuldade para deambular.

- Nutriçáo desequilibrada: menos que as necessidades corporais - caracterizada por: peso corporal $20 \%$ ou mais abaixo do ideal (IMC $<25$ ), inapetência, relato de ingesta menor de alimentos, dor abdominal. Relacionada à ausência total de dentes, dificuldade de mastigação, náuseas/ vômito, lesão em mucosa oral.

- Constipação - caracterizada por: dor abdominal, dor ao evacuar, fezes duras e secas, freqüência diminuída, ruídos hidroaéreos hipoativos. Relacionado à atividade física insuficiente (dificuldade para deambular, sedentarismo), à depressão/ tristeza, à obesidade, aos hábitos alimentares deficientes (nutrição diminuída), à desidratação.

- Eliminaçáo urinária prejudicada - caracterizada por: incontinência de urgência, disúria/anúria, nictúria. Relacionado ao dano sensório motor (diminuição da circulação cerebral) e múltiplas causas (doença de base).

- Disfunção sexual - caracterizada por: verbalização do problema (sente falta de ter relação sexual), 
limitações reais/percebidas impostas pela doença/ terapêutica, impotência. Relacionado à estrutura corporal da função alterada (amputação), falta de pessoa significativa (viuvez/divórcio).

- Padráo de sono perturbado - caracterizado por: insônia, dificuldade para dormir, sono interrompido. Relacionado à: depressão, solidáo, medo, dor, dispnéia.

- Deambulação prejudicada - caracterizada por: capacidade diminuída de deambular, subir escadas, andar em aclive/declive. Relacionado a requerer uso de aparelho ou artefato (bengala, muleta, andador, cadeira de rodas), totalmente dependente de terceiros (acamado).

- Angústia espiritual - caracterizada por: verbaliza estar separado de seu sistema de apoio (comunidade). Relacionada à mudança na vida, à doença crônica, à solidão/alienação social, à dor.

- Déficit no autocuidado para alimentar-se - caracterizado por: dificuldade para mastigar alimentos, preparar alimentos para ingestáo. Relacionado à dor, à ausência total de dentes, à dependência parcial/total de terceiros.

- Déficit no autocuidado para banho/higiene caracterizado por: incapacidade de lavar o corpo ou partes do corpo, secar o corpo, entrar e sair do banheiro. Relacionado à dor, ao prejuízo neuromuscular (diminuição da circulação cerebral), ao prejuízo musculoesquelético (amputação), à dependência parcial/total de terceiros.

- Déficit no autocuidado para higiene íntima caracterizado por: chegar ao vaso sanitário ou cadeira higiênica, sentar-se ou levantar-se do vaso sanitário ou cadeira higiênica. Relacionado à dor, ao prejuízo neuromuscular (diminuição da circulação cerebral), ao prejuízo musculoesquelético (amputação), à dependência parcial/ total de terceiros.

- Déficit no autocuidado para vestir-se/arrumar-secaracterizado por: colocar e tirar itens necessários de vestuários, calçar sapatos, meias, colocar roupas na parte inferior do corpo. Relacionado à dor, ao prejuízo neuromuscular (diminuição da circulação cerebral), ao prejuízo musculoesquelético (amputação), à dependência parcial/total de terceiros.

- Estilo de Vida - caracterizado por: rotina diária sem exercícios físicos (sedentarismo). Relacionado à falta de motivação/interesse (tristeza/ depressão).

- Conhecimento deficiente da patologia/terapêutica - caracterizado por: seguimento inadequado de instruçóes (analfabetismo), dependência de terceiros para medicar-se. Relacionado à falta de capacidade de recordar (desconhecimento, esquecimento).

- Dor - caracterizado por: evidência observada, distúrbio do sono (insônia), mudança no apetite e na alimentação (inapetência), estar acamado. Relacionado a varizes MMII, a lesôes teciduais, à amputação, à dispnéia, à disúria, ao edema.

- Medo - caracterizado por: produtividade e capacidade de aprendizagem diminuída (desconhecimento da patologia), estado de alerta aumentado (ansiedade). Relacionado à dor, à falta de familiaridade com experiência, à resposta aprendida (ansiedade).

- Distúrbio na imagem corporal - mudanças no envolvimento social, perda de parte do corpo (amputação), preocupaçáo com mudança ou perda (medo). Relacionado à doença, à cirurgia, ao trauma ou à lesão.

\section{Conclusão}

Acompanhando os processos de hospitalização do idoso portador de doença cardiovascular foi estudada uma populaçáo de 84 pacientes dentro da faixa etária de 60 anos ou mais em um hospital público na cidade de São Paulo. Os dados foram obtidos por meio da aplicaçáo de questionário (Histórico de Enfermagem) e exame físico céfalo-caudal. Os problemas de enfermagem encontrados foram organizados e analisados para o levantamento do diagnóstico específico de enfermagem.

A assistência ao idoso durante a hospitalização merece atenção de todos os profissionais que participam deste cuidado. Foi possível observar que um minucioso exame físico condiciona o enfermeiro ao levantamento correto do diagnóstico de enfermagem para que se possa ter a especifica intervenção. Esta representa uma das etapas que sustenta a Sistematização da Assistência de Enfermagem e a condiciona a melhora do quadro assistencial.

Com o levantamento dos diagnósticos de enfermagem específicos baseado em NANDA, foi possível visualizar o idoso como um todo, bem como a inter-relaçáo dos fatores que são causa e efeito de uma dada situação facilitando assim a elaboração de estratégias específicas a serem implementadas na assistência de enfermagem.

Levantar os problemas mais comuns dos idosos nos permite detectar as alteraçóes mais freqüentes 
que ocorrem nesta faixa etária. Assim, podemos dar-lhe uma assistência de qualidade e fazer as adaptaçóes necessárias de maneira mais efetiva, resolvendo a maior parte de seus problemas durante a intervenção, contribuindo para a redução do tempo de internação e melhoria da qualidade de vida.

\section{Referências}

1. Horta WA. A assistência de enfermagem ao adulto idoso. Rev Enferm Nov Dimens 1978;4(5):268-73.

2. Sá SPC, Ferreira MA. Cuidados fundamentais na arte de cuidar do idoso: uma questão para a enfermagem. Esc Anna Nery Rev Enferm 2004;8(1):46-52.

3. Papaléo MN, Carvalho ETF. Geriatria: fundamentos, clínica e terapêutica. 2a ed. São Paulo: Atheneu; 2005.

4. Campedelli MC. Consulta de enfermagem para o idoso: uma necessidade real. Rev Paul Enferm 1991;10(1):32-37.

5. Tier CG, Fontana RT, Soares NV. Refletindo sobre idosos institucionalizados. Rev Bras Enfermagem 2004; $57: 332-5$.

6. Estatuto do Idoso. Lei No. 10741 de 1 de outubro de 2003. Brasília: DOU; 2003.

7. Instituto Brasileiro de Geografia Estatística - IBGE. Dados Estatísticos - Envelhecimento Populacional. [online]. [citado 2006 Ago 08]. Disponível em: URL:http// www.ibge.gov.br

8. Diogo MJD, Ceolim MF, Cintra FA. Implantação do grupo de atenção à saúde do idoso (GRASI) no hospital de clínicas da Universidade Estadual de Campinas (SP): Relato de experiência. Rev. Latinoam Enfermagem 2000;8:85-90.
9. Jacob WF. Atividade física e envelhecimento saudável. São Paulo: Atheneu; 2006.

10. Tirado MGA. Princípios da avaliação gerontológica. In: $3^{\circ}$ Congresso Paulista de Geriatria e Gerontologia, 2003, Santos. Anais III GERP. Santos, 2003

11. IV Diretrizes Brasileiras de Hipertensão Arterial. Arq Bras Cardiol 2004;82(suppl 4):7-14.

12. Lainetti KR, Puma MG. Exame físico da pele do idoso: Um guia prático para o graduando de enfermagem e o enfermeiro [monografia]. Sáo Paulo: Universidade Cidade de São Paulo; 2002.

13. Taddei CFG, Ramos LR, Moraes JC, Wajngarten M, Libberman A, Santos SC, et al. Estudo multicêntrico de idosos atendidos em ambulatórios de cardiologia e geriatria de instituiçóes brasileiras Arq Bras Cardiol 1997;69:327-33.

14. Danzil NJ, Graziano KU. Como os enfermeiros coordenam os cuidados onde não há SAE. Cadernos Centro Universitário São Camilo 2001;4:79-90.

15. Horta WA. Processo de enfermagem. São Paulo: EPU; 1979.

16. Paula JC, Cintra FA. A relevância do exame físico do idoso para a assistência de enfermagem hospitalar. Acta Paul Enfermagem 2005;18:301-6.

17. Lourenço RA, Martins CSF, Sanchez MAS, Veras RP. Assistência ambulatorial geriátrica: hierarquização da demanda. Rev Saúde Pública 2005;39:311-8.

18. Farias JN. Diagnósticos de enfermagem: uma abordagem conceitual e prática. João Pessoa: Santa Marta; 1990.

19. North American Nursing Diagnosis Association. Diagnósticos de enfermagem da NANDA: definiçóes e classificaçóes 2005 - 2006. Porto Alegre: Artmed; 2006.

20. Duarte YAO. A influência da formação acadêmica do enfermeiro na assistência ao Idoso. Âmbito Hosp 1994;6:61-6. 